\title{
Detection of incidental colorectal pathology on positron emission tomography/computed tomography (PET/CT)
}

Milton Mui ${ }^{1}$ MBBS, BMedSci

Timothy Akhurst ${ }^{2}$ MD, MBBS, FRACP

Satish K. Warrier ${ }^{1}$ MS, FRACS

A. Craig Lynch $^{1}$ MMedSci, FRACS, FCSSANZ

Alexander G. Heriot ${ }^{1}$ MD, MBA, FRACS, FRCS

${ }^{1}$ Department of Cancer Surgery, Peter MacCallum Cancer Centre, East Melbourne, Victoria, Australia.

${ }^{2}$ Department of Cancer Imaging, Peter MacCallum Cancer Centre, East Melbourne, Victoria, Australia.

Running head

Detection of incidental colorectal pathology on PET/CT

Keywords

Incidental Findings, Colorectal Neoplasms, Fluorodeoxyglucose F18, Positron-Emission Tomography, Colonoscopy

Content

No. of tables included: 3

Word count: 237 (abstract); 2288 (text)

Corresponding author

Dr Milton Mui

Department of Cancer Surgery

Peter MacCallum Cancer Centre

2 St Andrews Place

East Melbourne, VIC 3002

Australia

This is the author manuscript accepted for publication and has undergone full peer review but has not been through the copyediting, typesetting, pagination and proofreading process, which may lead to differences between this version and the Version of Record. Please cite this article as doi: 10.1111/ans.13739

This article is protected by copyright. All rights reserved. 
Email:mch.mui@gmail.com

*Corresponding author is not a recipient of a research scholarship

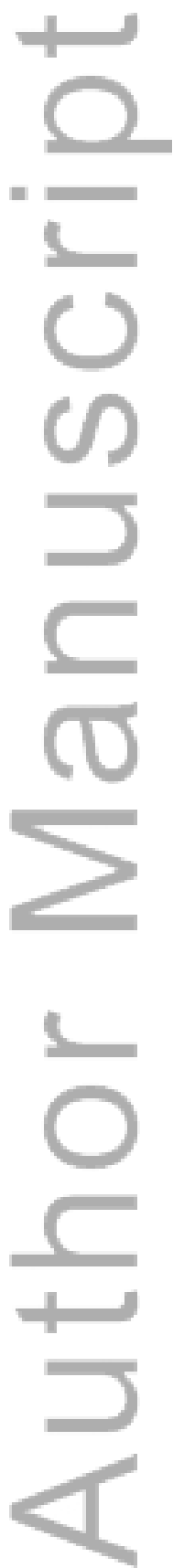

This article is protected by copyright. All rights reserved. 


\section{Abstract}

\section{Introduction:}

Positron emission tomography/computed tomography (PET/CT) is an important modality in cancer imaging.

With its increasing availability and use, it is not uncommon to detect incidental focal colorectal ${ }^{18} \mathrm{~F}$-FDG uptake which poses a diagnostic challenge, as they may be associated with malignant or pre-malignant colorectal lesions. The aim of our study is to determine the proportion of these findings which represents true pathology.

\section{Methods and Materials:}

Patients with incidental focal colorectal ${ }^{18}$ F-FDG uptake on PET/CT who subsequently underwent colonoscopy between January 2002 to September 2013 were identified from a prospective database in a tertiary referral centre. PET/CT results were correlated with colonoscopy and pathology results in these patients. Positive predictive values (PPVs) and 95\% confidence intervals (CIs) of PET/CT in the detection of incidental colorectal pathology were calculated.

\section{Results:}

One hundred and forty-eight patients (92 males and 56 females), with a mean age 73 years (range of 36 to 93 years) were included in the study. A total of 170 foci of colorectal ${ }^{18} \mathrm{~F}-\mathrm{FDG}$ uptake were detected on PET/CT. Of these, 101 foci corresponded to a malignant or pre-malignant lesion (PPV 59\%; 95\% CI: 52-67\%). On a perpatient analysis, 93 patients had at least one focus of colorectal ${ }^{18} \mathrm{~F}$-FDG uptake which corresponded to a premalignant or malignant lesion (PPV 63\%; 95\% CI: 54-71\%).

\section{Conclusion:}

Focal colorectal ${ }^{18} \mathrm{~F}$-FDG uptake on PET/CT is associated with a significant proportion of malignant or premalignant lesions. Further evaluation with colonoscopy is recommended.

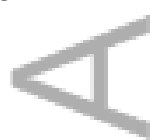




\section{Introduction}

For over two decades, positron emission tomography with 18-fluoro-2-deoxyglucose ( ${ }^{18}$ FDG-PET) has been used in the staging and monitoring of various cancers, including non-small cell lung cancers, lymphomas, melanomas, and head and neck cancers (1). Combined with the anatomical data provided by computed tomography (CT), PET/CT has an improved diagnostic accuracy and is now considered an important modality in cancer imaging (2).

With its increasing availability and use in cancer patients, it is not uncommon to detect incidental ${ }^{18}$ F-FDG uptake on PET/CT in sites other than where the primary cancer is known or suspected, posing a diagnostic challenge to clinicians. A common site is the large bowel, where the prevalence of incidental focal colorectal ${ }^{18}$ F-FDG uptake detected by PET or PET/CT is estimated at $3.6 \%$ (3). While diffuse and segmental patterns of ${ }^{18}$ F-FDG uptake are attributed to benign or inflammatory causes, focal uptake is associated with a significant risk of neoplasia (4-11). Given that colorectal cancer may be insidious in onset and run a silent course until late, these radiological findings should not be ignored (12). Prompt diagnosis may have a significant impact on patient outcome, particularly in these patients who already has a pre-existing cancer as it may necessitate a management plan that integrates treatment for both cancers within a relatively short period of time.

Previous studies have reported large numbers of patients who had incidental focal colorectal ${ }^{18}$ F-FDG uptake on PET or PET/CT, but few had sufficient numbers who subsequently underwent colonoscopy to investigate the underlying pathology. Therefore, the aim of our study is to determine the proportion of incidental focal colorectal ${ }^{18}$ F-FDG uptake on PET/CT which represents true pathology.

\section{Materials and Methods}

\section{Study population}

Patients with incidental focal colorectal ${ }^{18} \mathrm{~F}$-FDG uptake on PET/CT who subsequently underwent colonoscopy between January 2002 and September 2013 were identified from a prospective database in a tertiary referral centre. Focal colorectal ${ }^{18}$ F-FDG uptake is termed incidental if it is detected in the large bowel on PET/CT in 
patients who have a non-colorectal or unknown primary cancer. The institutional review board approved the study and waived the need for individual patient consent for reviewing imaging studies and electronic medical records.

\section{PET/CT protocol}

All patients were instructed to fast for at least 6 hours before administration of ${ }^{18} \mathrm{~F}-\mathrm{FDG}(5 \mathrm{MBq} / \mathrm{kg}$, up to max. $400 \mathrm{MBq})$. After resting for at least 60 minutes for 18-FDG uptake to occur, PET/CT scans were performed from the level of skull base to upper thighs using one of the dedicated PET/CT scanners available at our institution (Discovery LS PET/4-slice helical CT, Discovery STE/8-slice helical CT or Discovery 690/64-slice helical CT, General Electric Medical Systems, Milwaukee, WI; Siemens Biograph 64 slice PET/CT, Siemens Healthcare, Erlangen, Germany).

Emission data were processed by iterative reconstruction both with and without attenuation correction using the non-contrast CT scan obtained. All CT, PET and PET/CT images were displayed simultaneously on a Siemens Multimodality Workstation. An experienced nuclear medicine physician generated a clinical report after reviewing these images, previous imaging results and clinical information to reflect routine clinical practice.

\section{Colonoscopy}

Colonoscopy was performed by consultant colorectal surgeons who were accredited by Gastroenterological Society of Australia (GESA). All patients received standard bowel preparation on the day before the procedure. Colonoscopy was passed to the caecum as confirmed by the ileocaecal valve. Any abnormalities identified on colonoscopy were recorded, with biopsies taken where appropriate.

\section{Data analysis}

In all patients, PET/CT results were correlated with colonoscopy \pm pathology results. Both per-lesion and perpatient analyses were then performed.

On a per-lesion analysis, a PET/CT finding was considered a 'true positive' if a focus of colorectal ${ }^{18} \mathrm{~F}-\mathrm{FDG}$ uptake on PET/CT corresponded to a structural abnormality identified in the same or adjacent segment on 
colonoscopy. A PET/CT finding was considered 'false positive' if a focus of colorectal ${ }^{18} \mathrm{~F}$-FDG uptake on PET/CT did not correspond to a structural abnormality identified in the same or adjacent segment on colonoscopy. On a per-patient analysis, a PET/CT exam was considered 'true positive' if a patient had a positive PET/CT exam and e 1 structural abnormalities identified on colonoscopy. A PET/CT exam was considered 'false positive' if a patient had a positive PET/CT exam but no structural abnormalities identified on colonoscopy.

\section{Statistical Analysis}

Positive predictive values (PPVs) of PET/CT in the detection of incidental colorectal pathology were calculated using standard formulas, both on a per-lesion and per-patient level. Results are expressed as percentages where appropriate. A 95\% confidence interval (CI) is determined for each parameter.

\section{Results}

\section{Patient characteristics}

A total of 148 patients with incidental focal colorectal FDG uptake were included in the study. There were 92 males and 56 females with a mean age of 73 years (range of 36 to 93 years). The indications for PET/CT are shown in Table 1.

\section{PET/CT results}

There were a total of 170 foci of colorectal ${ }^{18} \mathrm{~F}-\mathrm{FDG}$ uptake detected on PET/CT. One hundred and thirty patients had a single focus of colorectal ${ }^{18} \mathrm{~F}-\mathrm{FDG}$ uptake, 15 patients had two foci, 2 patients had three foci, and 1 patient had four foci. The distribution of the foci on PET/CT was as follows: ascending colon $(n=31)$, transverse colon $(n=13)$, descending colon $(n=21)$, rectosigmoid colon $(n=102)$, anorectal region $(n=3)$.

\section{Colonoscopy results}

The mean time interval between the detection of focal colorectal ${ }^{18} \mathrm{~F}$-FDG uptake on PET/CT and subsequent colonoscopy was 52 days (range of 4 to 385 days). 
A total of 212 lesions were identified on colonoscopy. The distribution of the colorectal lesions was as follows: ascending colon $(n=44)$, transverse colon $(n=17)$, descending colon $(n=16)$, rectosigmoid colon $(n=134)$, anorectal region $(\mathrm{n}=1)$. The histology results are shown in Table 2.

\section{Per-lesion analysis}

Of the 170 foci of colorectal ${ }^{18} \mathrm{~F}$-FDG uptake, 120 corresponded to a structural abnormality identified on colonoscopy (PPV 71\%, 95\% CI: 63-77\%). The distribution of the foci on PET/CT with their corresponding pathology are shown in Table 3. Importantly, 101/170 foci corresponded to a pre-malignant or malignant lesion (PPV 59\%, 95\% CI: 52-67\%). These included 20 adenocarcinomas, 20 adenomas with high grade dysplasia, and 61 adenomas with low grade dysplasia.

Three lesions were inflammatory in nature: proctitis (2) and sigmoid diverticulitis (1). Eight lesions were benign: hyperplastic polyps (3), myoglandular polyp (1), fibroepithelial polyp (1), hamartoma (1), polypoid epithelial hyperplasia (1), benign-looking tumour with no biopsy taken (1). Four lesions had normal histology on biopsy. Four lesions were not biopsied on colonoscopy.

The PPVs in the detection of pre-malignant or malignant lesions in the different segments of colon were as follows: $61 \%(95 \% \mathrm{CI}: 42-78 \%)$ in the ascending colon, $31 \%(95 \% \mathrm{CI}: 10-61 \%)$ in the transverse colon, $29 \%$ (95\% CI: $12-52 \%)$ in the descending colon, $70 \%$ (95\% CI: $60-78 \%)$ in the rectosigmoid colon, and $33 \%(95 \%$ CI: $1-87 \%$ ) in the anorectal region.

\section{Per-patient analysis}

Among the 148 patients who underwent colonoscopy, 116 patients had abnormalities identified on colonoscopy (PPV 78\%; 95\% CI: 71-85\%).

One hundred and five patients had at least one focus of colorectal ${ }^{18} \mathrm{~F}-\mathrm{FDG}$ uptake which corresponded to an abnormality identified on colonoscopy (PPV 71\%, 95\% CI: 63-78\%). Of those, 93 harboured at least one premalignant or malignant lesion (PPV 63\%, 95\% CI: 54-71\%), with 12 patients having two or more pathologies. 


\section{Discordant findings}

On a per-lesion analysis, 50 foci of colorectal ${ }^{18} \mathrm{~F}-\mathrm{FDG}$ uptake on PET/CT did not correspond to any structural abnormality identified in the same or adjacent segment on colonoscopy and were considered 'false positives'. On a per-patient analysis, $34 \mathrm{PET} / \mathrm{CT}$ exams did not have abnormalities identified on colonoscopy and were considered 'false positives'.

Of the 212 lesions identified on colonoscopy, 92 (43\%) did not correspond to any ${ }^{18} \mathrm{~F}-\mathrm{FDG}$ uptake on PET/CT: adenocarcinomas (2), adenomas (43), benign lesions (18), normal lesions (6), and lesions of unknown histology (23).

\section{Discussion}

Our study showed that $120 / 170$ (71\%) foci of colorectal ${ }^{18}$ F-FDG uptake on PET/CT corresponded to a structural abnormality identified on colonoscopy. Of these, 101 (59\%) turned out to be pre-malignant or malignant lesions on biopsy. Similar per-patient findings were obtained, with 93/148 patients (63\%) having one or more biopsy-proven pre-malignant or malignant lesions. These results are consistent with those of previous studies where the PPV of focal colorectal ${ }^{18}$ F-FDG uptake on PET/CT in detecting a malignant or pre-malignant colorectal lesion is estimated to be between 50 and $80 \%(5-7,9)$. Therefore, it adds weight to current guidelines that prompt evaluation with colonoscopy is warranted. Early detection of second primary tumour has been shown to improve overall outcome (13), and removal of polyps can result in reduction in incidence and mortality from colorectal cancer (14).

It should be noted that among the 19 foci of colorectal ${ }^{18} \mathrm{~F}-\mathrm{FDG}$ uptake on PET/CT which were initially classified as non-malignant, 1 was subsequently proven to be adenocarcinoma post-colectomy and 1 was an adenoma with low-grade dysplasia on repeat colonoscopy. The inflammatory lesions were presumably FDGavid on PET/CT due to the increased metabolism of white cells (15) but it is unclear why benign lesions were FDG-avid as well, although in the case of hyperplastic polyps, it has been suggested that it may be related to their proliferative activity (16). It is also important to consider that some of these structural abnormalities 
identified on colonoscopy may have been incidental findings in the context of a 'false positive' PET/CT result, given that there is a margin of error in correlating the exact site between the two.

Few studies have evaluated the likelihood of pathology based on the site of focal ${ }^{18}$ F-FDG uptake on PET/CT (8, 9). Our study showed that there may a predilection for the ascending and rectosigmoid colon, although it is unclear why this is the case. A study by Lee et al. has found that focal ${ }^{18} \mathrm{~F}-\mathrm{FDG}$ uptake in the proximal colon was associated with a high likelihood of pathology (9). However, these findings are in contrast to those reported by Peng et al. where FDG uptake in the ascending colon was an independent negative predictor of finding cancer or polyps (8). Therefore, the site of focal ${ }^{18} \mathrm{~F}-\mathrm{FDG}$ uptake on PET/CT should not be used as a guide to determine the significance of these findings until further studies are available.

There were a total of 50 foci of colorectal ${ }^{18} \mathrm{~F}$-FDG uptake on PET/CT which did not correspond to an abnormality identified on colonoscopy (FDR 29\%, 95\% CI: 23-37\%). This may be due to several reasons, including physiological uptake by smooth muscle, bowel peristalsis and constipation (17). In addition, inflammation (e.g. inflammatory bowel disease, colitis) had been shown to cause increased colorectal F18-FDG uptake on PET/CT, although it is usually diffuse or segmental in nature $(18,19)$. It is also possible that some patients had inflammation which settled prior to colonoscopy. Finally, colonoscopic abnormalities may have been missed due to presence of submucosal lesions, difficult procedure or inadequate bowel preparation.

Conversely, 92 lesions (43\%) did not correspond to any ${ }^{18} \mathrm{~F}-\mathrm{FDG}$ uptake on PET/CT, including 45 premalignant and malignant lesions. This is to be expected in the case of benign lesions but is not entirely surprising either in the case of pre-malignant and malignant lesions, given that PET/CT is not a primary screening tool for colorectal cancer due to its poor sensitivity in detecting adenomas (20). A range of factors may be involved, including the spatial resolution of the PET/CT scanner, bowel peristalsis and lesion size. It has been shown that the sensitivity of PET/CT in detecting adenomas improves with increasing size.

Our study included 148 patients with correlation of PET/CT and pathology results in a single institution, making it one of the largest studies to date to establish diagnosis and assess the significance of incidental focal colorectal ${ }^{18} \mathrm{~F}-\mathrm{FDG}$ uptake on PET/CT. Previous studies have large sample sizes to look at the prevalence of 
incidental focal colorectal ${ }^{18}$ F-FDG uptake on PET/CT, but few had sufficient numbers who subsequently underwent colonoscopy $(8,10,11)$. In addition, most patients in our study underwent colonoscopy within the first 2 months of PET/CT findings, hence an accurate histological diagnosis was obtained. Finally, our institution maintained a centralised electronic medical records system since the early 2000s, which allowed us to comprehensively review all patient history, imaging results, colonoscopy reports and pathology results.

There are several limitations to this study. Firstly, our study did not include patients with incidental focal colorectal ${ }^{18} \mathrm{~F}$-FDG uptake on PET/CT who failed to undergo colonoscopy due to various reasons (e.g. patient refusal, palliative intent, multiple co-morbidities), thus affecting the true accuracy of PET/CT. However, it could be argued that this would reflect routine clinical practice. Accordingly, we were unable to determine the specificity and negative predictive values of PET/CT in detecting incidental colorectal pathology. Secondly, some patients may have undergone CT colonography instead, although this number is unlikely to be significant given that it is not the first-line investigation for the detection of colorectal pathology. Thirdly, although colonoscopy remains the gold standard in detecting colorectal pathology, it has a miss rate of $22 \%$ for polyps of any size (21). Therefore, misclassification bias may have been introduced.

At present, colonoscopy is still the reference standard to detect true colorectal pathology. Although PET/CT colonography has been proposed to improve accuracy in diagnosing colorectal lesions, it is still in experimental stages and the metabolic information from PET does not appear to increase the accuracy of CT colonography (22). However, it may be valuable in patients who has an obstructing tumour and a complete colonoscopy cannot be performed (23), as well as those who have contraindications or refuse colonoscopy. Further efforts should be made to evaluate the usefulness of PET/CT colonography, as well as develop more sensitive detectors and tumour-specific radiotracers to improve accuracy of PET/CT in detecting pre-malignant or malignant colorectal lesions.

\section{Conclusion}

Focal colorectal ${ }^{18}$ F-FDG uptake on PET/CT is associated with a significant risk of pre-malignant or malignant lesions and may affect subsequent management. Further evaluation with colonoscopy is recommended. 


\section{Disclosure statement}

The authors declare no potential conflict of interest.

\section{References}

1. Juweid ME, Cheson BD. Positron-emission tomography and assessment of cancer therapy. The New England journal of medicine. 2006;354(5):496-507.

2. Bar-Shalom R, Yefremov N, Guralnik L, Gaitini D, Frenkel A, Kuten A, et al. Clinical performance of $\mathrm{PET} / \mathrm{CT}$ in evaluation of cancer: additional value for diagnostic imaging and patient management. J Nucl Med. 2003;44(8):1200-9.

3. Treglia G, Taralli S, Salsano M, Muoio B, Sadeghi R, Giovanella L. Prevalence and malignancy risk of focal colorectal incidental uptake detected by (18)F-FDG-PET or PET/CT: a meta-analysis. Radiol Oncol. 2014;48(2):99-104.

4. Tatlidil R, Jadvar H, Bading JR, Conti PS. Incidental colonic fluorodeoxyglucose uptake: correlation with colonoscopic and histopathologic findings. Radiology. 2002;224(3):783-7.

5. Kamel EM, Thumshirn M, Truninger K, Schiesser M, Fried M, Padberg B, et al. Significance of incidental 18F-FDG accumulations in the gastrointestinal tract in PET/CT: correlation with endoscopic and histopathologic results. J Nucl Med. 2004;45(11):1804-10.

6. Gutman F, Alberini JL, Wartski M, Vilain D, Le Stanc E, Sarandi F, et al. Incidental colonic focal lesions detected by FDG PET/CT. AJR Am J Roentgenol. 2005;185(2):495-500.

7. Israel O, Yefremov N, Bar-Shalom R, Kagana O, Frenkel A, Keidar Z, et al. PET/CT detection of unexpected gastrointestinal foci of $18 \mathrm{~F}-\mathrm{FDG}$ uptake: incidence, localization patterns, and clinical significance. J Nucl Med. 2005;46(5):758-62.

8. Peng J, He Y, Xu J, Sheng J, Cai S, Zhang Z. Detection of incidental colorectal tumours with 18Flabelled 2-fluoro-2-deoxyglucose positron emission tomography/computed tomography scans: results of a prospective study. Colorectal Dis. 2011;13(11):e374-8.

9. Lee JC, Hartnett GF, Hughes BG, Ravi Kumar AS. The segmental distribution and clinical significance of colorectal fluorodeoxyglucose uptake incidentally detected on PET-CT. Nucl Med Commun. 2009;30(5):3337 .

10. Liu T, Behr S, Khan S, Osterhoff R, Aparici CM. Focal Colonic FDG Activity with PET/CT: Guidelines for Recommendation of Colonoscopy. World J Nucl Med. 2015;14(1):25-30.

11. Gollub MJ, Grewal RK, Panu N, Thipphavong S, Sohn M, Zheng J, et al. Diagnostic accuracy of (1)(8)F-FDG PET/CT for detection of advanced colorectal adenoma. Clin Radiol. 2014;69(6):611-8.

12. Lieberman DA, Weiss DG, Bond JH, Ahnen DJ, Garewal H, Chejfec G. Use of colonoscopy to screen asymptomatic adults for colorectal cancer. Veterans Affairs Cooperative Study Group 380. The New England journal of medicine. 2000;343(3):162-8. 
13. Choy AT, van Hasselt CA, Chisholm EM, Williams SR, King WW, Li AK. Multiple primary cancers in Hong Kong Chinese patients with squamous cell cancer of the head or neck. Cancer. 1992;70(4):815-20.

14. Winawer SJ, Zauber AG, Ho MN, O'Brien MJ, Gottlieb LS, Sternberg SS, et al. Prevention of colorectal cancer by colonoscopic polypectomy. The National Polyp Study Workgroup. The New England journal of medicine. 1993;329(27):1977-81.

15. Cook GJ, Fogelman I, Maisey MN. Normal physiological and benign pathological variants of 18fluoro-2-deoxyglucose positron-emission tomography scanning: potential for error in interpretation. Semin Nucl Med. 1996;26(4):308-14.

16. Wong WM, Mandir N, Goodlad RA, Wong BC, Garcia SB, Lam SK, et al. Histogenesis of human colorectal adenomas and hyperplastic polyps: the role of cell proliferation and crypt fission. Gut. 2002;50(2):212-7.

17. Shreve PD, Anzai Y, Wahl RL. Pitfalls in oncologic diagnosis with FDG PET imaging: physiologic and benign variants. Radiographics. 1999;19(1):61-77; quiz 150-1.

18. Kresnik E, Gallowitsch HJ, Mikosch P, Wurtz F, Alberer D, Hebenstreit A, et al. (18)F-FDG positron emission tomography in the early diagnosis of enterocolitis: preliminary results. Eur J Nucl Med Mol Imaging. 2002;29(10):1389-92.

19. Neurath MF, Vehling D, Schunk K, Holtmann M, Brockmann H, Helisch A, et al. Noninvasive assessment of Crohn's disease activity: a comparison of 18F-fluorodeoxyglucose positron emission tomography, hydromagnetic resonance imaging, and granulocyte scintigraphy with labeled antibodies. The American journal of gastroenterology. 2002;97(8):1978-85.

20. Yasuda S, Fujii H, Nakahara T, Nishiumi N, Takahashi W, Ide M, et al. 18F-FDG PET detection of colonic adenomas. J Nucl Med. 2001;42(7):989-92.

21. van Rijn JC, Reitsma JB, Stoker J, Bossuyt PM, van Deventer SJ, Dekker E. Polyp miss rate determined by tandem colonoscopy: a systematic review. The American journal of gastroenterology. 2006;101(2):343-50.

22. Mainenti PP, Salvatore B, D'Antonio D, De Falco T, De Palma GD, D'Armiento FP, et al. PET/CT colonography in patients with colorectal polyps: a feasibility study. Eur J Nucl Med Mol Imaging. 2007;34(10):1594-603.

23. Nagata K, Ota Y, Okawa T, Endo S, Kudo SE. PET/CT colonography for the preoperative evaluation of the colon proximal to the obstructive colorectal cancer. Diseases of the colon and rectum. 2008;51(6):882-90. 


\section{Tables}

Table 1. Types of primary cancer in patient population.

\begin{tabular}{lll}
\hline Primary cancer & Number of patients & \% \\
Head \& neck cancer & 28 & 18.9 \\
Melanoma & 24 & 16.2 \\
Lymphoma & 22 & 14.9 \\
Lung cancer & 19 & 12.8 \\
Gynaecological cancer & 17 & 11.5 \\
Soft tissue and bone cancer & 10 & 6.8 \\
Upper GI cancer & 9 & 6.1 \\
Cutaneous SCC & 6 & 4.1 \\
Unknown primary & 6 & 4.1 \\
Others & 7 & 4.7 \\
Total & $\mathbf{1 4 8}$ & \\
\hline & & \\
& & \\
& & \\
& & \\
\end{tabular}

This article is protected by copyright. All rights reserved. 
Table 2. Distribution and histology results of lesions identified on colonoscopy.

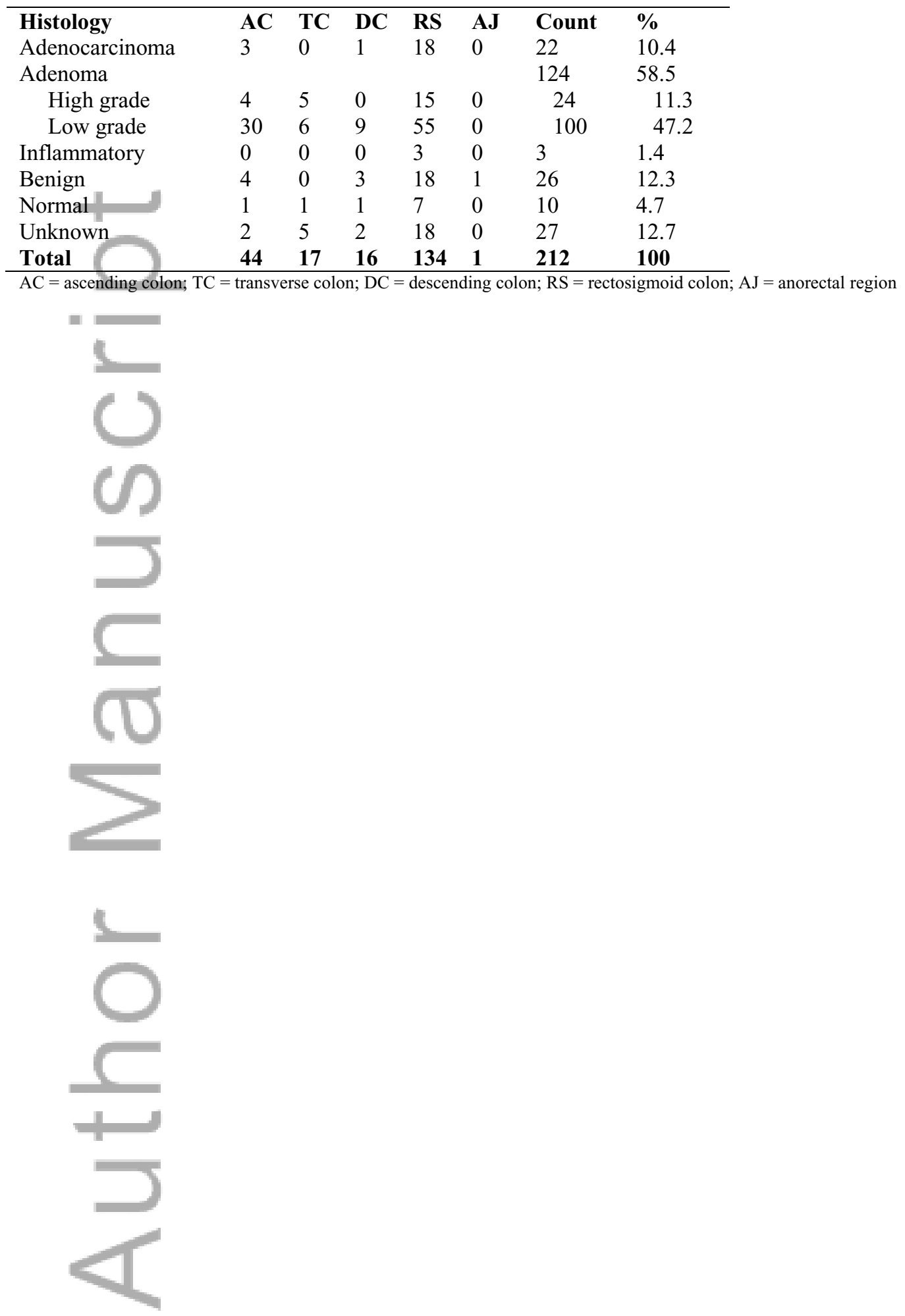

This article is protected by copyright. All rights reserved. 
Table 3. Distribution and histology results of focal colorectal ${ }^{18}$ F-FDG uptake on PET/CT

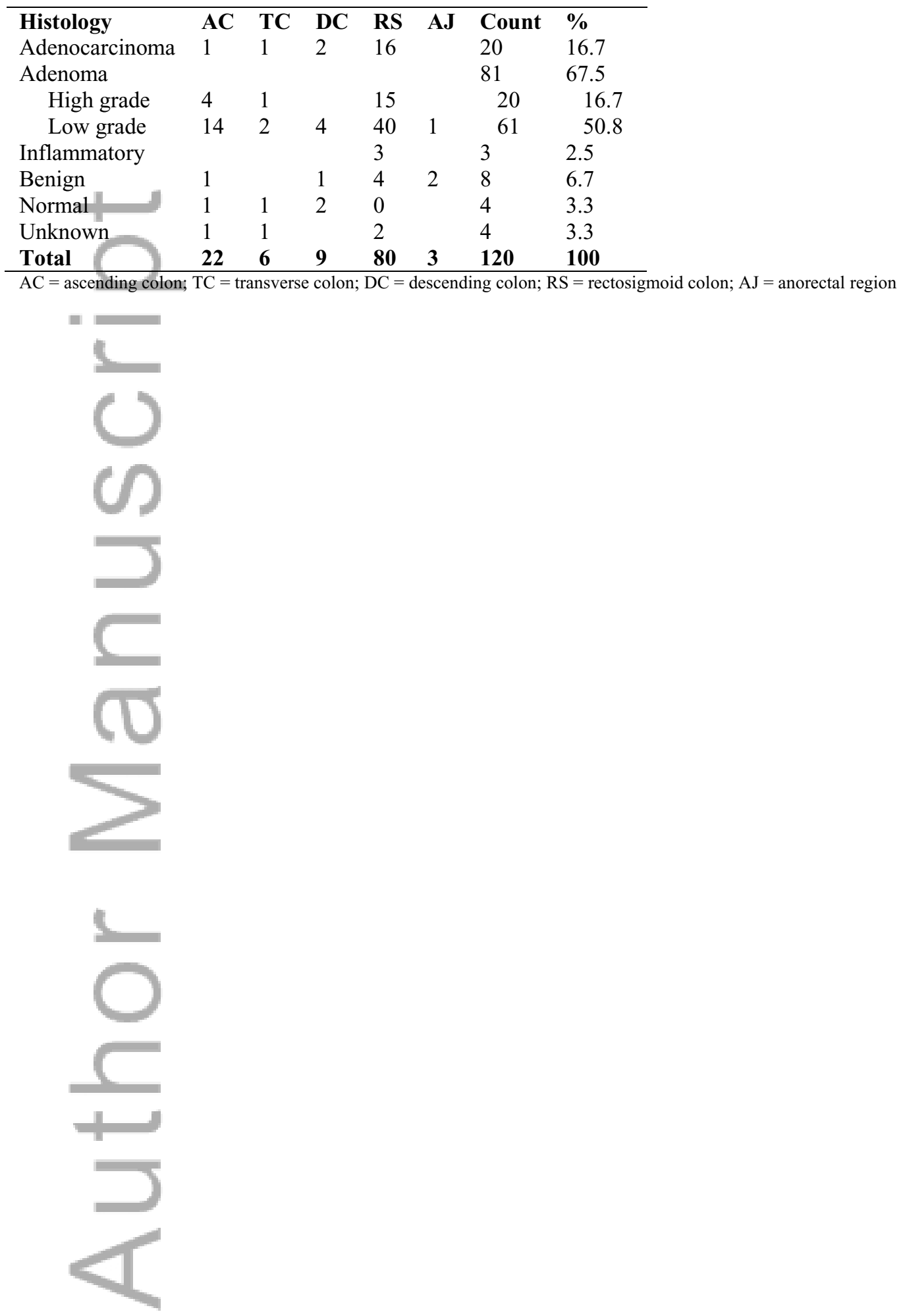

This article is protected by copyright. All rights reserved. 


\section{University Library}

\section{- M M N E R VA A gateway to Melbourne's research publications}

Minerva Access is the Institutional Repository of The University of Melbourne

Author/s:

Mui, M;Akhurst, T;Warrier, SK;Lynch, AC;Heriot, AG

Title:

Detection of incidental colorectal pathology on positron emission tomography/computed tomography

Date:

2018-03-01

Citation:

Mui, M., Akhurst, T., Warrier, S. K., Lynch, A. C. \& Heriot, A. G. (2018). Detection of incidental colorectal pathology on positron emission tomography/computed tomography. ANZ JOURNAL OF SURGERY, 88 (3), pp.E122-E126. https://doi.org/10.1111/ans.13739.

Persistent Link:

http://hdl.handle.net/11343/291667 